\title{
Ferromagnetic semiconductor InMnAs layers grown by pulsed laser deposition on GaAs
}

\author{
Yu A Danilov ${ }^{1}$, A V Kudrin ${ }^{1}$, O V Vikhrova ${ }^{1}$, B N Zvonkov ${ }^{1}$, \\ Yu N Drozdov ${ }^{2}$, M V Sapozhnikov ${ }^{2}$, S Nicolodi ${ }^{3}$, E R Zhiteytsev ${ }^{4}$, \\ N M Santos ${ }^{4}$, M C Carmo ${ }^{4}$ and N A Sobolev ${ }^{4}$ \\ ${ }^{1}$ Physico-Technical Research Institute of Nizhny Novgorod State University, Gagarine Av. 32/3, 603950 \\ Nizhny Novgorod, Russia \\ ${ }^{2}$ Institute for Physics of Microstructures, RAS, 603950 Nizhny Novgorod, Russia \\ ${ }^{3}$ Instituto de Física, UFRGS, Av. Bento Gonçalves 9500, 91501-970 Porto Alegre, RS, Brazil \\ ${ }^{4}$ Departamento de Física and I3N, Universidade de Aveiro, 3810-193 Aveiro, Portugal \\ E-mail: danilov@nifti.unn.ru
}

Received 23 July 2008, in final form 25 November 2008

Published 9 January 2009

Online at stacks.iop.org/JPhysD/42/035006

\begin{abstract}
InMnAs layers were grown in a quartz reactor by YAG : Nd pulsed laser ablation of solid targets (InAs and Mn) in hydrogen and arsine flow. The crystal quality and the phase composition were analysed by $\mathrm{x}$-ray diffraction. The electrical properties were derived from the Hall effect measurements. The InMnAs magneto-optical and magnetic properties were studied by means of the Kerr effect, alternating gradient field magnetometry and ferromagnetic (FM) resonance measurements. The dependence of the electrical and magnetic properties of the layers on the Mn content was investigated. The InMnAs layers exhibit FM properties at temperatures at least up to $300 \mathrm{~K}$.
\end{abstract}

\section{Introduction}

Ferromagnetic (FM) properties of highly Mn-doped InAs were established in 1989 [1]. Up to now the main technique of Mndoped III-V semiconductor growth remains low-temperature molecular-beam epitaxy (LT-MBE). The properties of InMnAs layers grown by this method essentially depend on the process temperature $\left(T_{\mathrm{g}}\right)$ and $\mathrm{Mn}$ concentration.

The $\mathrm{p}-\mathrm{In}_{1-x} \mathrm{Mn}_{x}$ As layers, obtained at $275^{\circ} \mathrm{C}$ and $x \approx$ 0.013 by LT-MBE, demonstrated nonlinear magnetic-field dependences of the Hall resistance with a hysteresis loop [2]. According to the temperature dependences of magnetization and Hall resistance, in this case the FM-paramagnetic transition point (Curie temperature, $T_{\mathrm{C}}$ ) was $7.5 \mathrm{~K}$. These InMnAs layers with a relatively low $\mathrm{Mn}$ content were single phase. Considering that ferromagnetism in (III, Mn) V semiconductors arises from interaction of holes with magnetic moments of $\mathrm{Mn}$ ions, it is advisable to enhance the Mn content to increase the Curie temperature. However, in compliance with the phase diagram of InMnAs in MBE growth [3], segregation of solid solution of $\mathrm{Mn}$ in InAs and formation of MnAs clusters were observed for $x>0.03$ and $T_{\mathrm{g}}$ in the range between 270 and $375^{\circ} \mathrm{C}$. A decrease in the $T_{\mathrm{g}}$ value to $160-250^{\circ} \mathrm{C}$ suppresses the MnAs phase formation, but causes $\mathrm{p}-\mathrm{n}$ conversion of conductivity type and, as a result, the disappearance of FM properties. An implementation of $(\mathrm{Al}, \mathrm{Ga}) \mathrm{Sb}$ and InAs/(Al, Ga)Sb buffer layers and use of $x$ between 0.06 and 0.10 at the growth temperature $\approx 250{ }^{\circ} \mathrm{C}$ provided a way for the formation of p-type InMnAs layers with $T_{\mathrm{C}}=30-50 \mathrm{~K}[3,4]$. The highest Curie temperature $(\sim 90 \mathrm{~K})$ for the MBE technique was obtained at $x \approx 0.12$ and with the use of the $\mathrm{Al}(\mathrm{As}, \mathrm{Sb})$ buffer layer for InMnAs growth in excess of As with the following thermal low-temperature annealing [5].

An alternative method of InMnAs formation is metalorganic chemical vapour (MOCVD) epitaxy at $T_{\mathrm{g}}=460$ $530{ }^{\circ} \mathrm{C}[6-8]$. The authors reasoned that the InMnAs layers, obtained by this technique, were free from inclusions of the MnAs phase for Mn concentrations up to $x=0.14$. Under magnetization measurements, these layers displayed Curie temperature $\sim 330 \mathrm{~K}$. In the author's opinion, the mechanism of room-temperature ferromagnetism in such layers was 
associated with effects of short-range order in $\mathrm{Mn}$ atom arrangement and the formation of $\mathrm{Mn}-\mathrm{Mn}$ dimers in the crystal lattice of InAs [8,9]. It is of primary importance that these MOCVD InMnAs layers exhibited only an ordinary Hall effect in the temperature range $78-300 \mathrm{~K}[10]$.

For InMnAs deposition a technique of laser ablation in vacuum was also used [11]. The layers, deposited at $450{ }^{\circ} \mathrm{C}$ with $\mathrm{Mn}$ content of $\sim 10$ at $\%$ on i-GaAs (100) substrates, had $n$-type conductivity. Conductivity conversion to p-type and anomalous Hall effect (AHE) were observed only after annealing the layers by $25 \mathrm{~ns}$ ruby laser pulses. An estimation of Curie temperature for these layers provided the value of $40 \mathrm{~K}[11]$.

Taking into consideration some remarkable properties of LT-MBE grown InMnAs structures (in particular, lightinduced ferromagnetism [12] and control of ferromagnetism by electric field [13]), a search for new methods and best conditions of InMnAs layer formation is important. This point is also important because for many applications of FM semiconductors the Curie temperature must be above or at least equal to room temperature (RT).

In this work, the InMnAs layers were grown by the pulsed laser ablation technique developed, as concerns the application to (III,Mn)V semiconductors, by us [14]. Our deposition method is distinct from the technique, used in [11], by the presence of a gaseous medium with a pressure of $\approx 25-50$ Torr in the way of sputtered particles from the target to the substrate. This condition causes decrease in the energy of the particles, collided with the substrate, and a corresponding reduction in defect formation in the grown layer [14]. The InMnAs layers exhibit FM properties at temperatures at least up to $300 \mathrm{~K}$.

\section{Experimental techniques}

The InMnAs layers were grown in a quartz reactor by pulsed laser ablation of solid targets in a hydrogen and arsine flow. We used a $Q$-switched YAG: Nd laser $(\lambda=1.06 \mu \mathrm{m})$ to sputter the wafer targets made of $\mathrm{Mn}$ and monocrystal InAs $\left(p \sim 10^{17} \mathrm{~cm}^{-3}\right.$ ). The description of the technique, including the geometry of the ablation chamber, has been detailed in [14]. The quantity of Mn (controlled by the sputtering time ratio of Mn and InAs, $\left.Y_{\mathrm{Mn}}=t_{\mathrm{Mn}} /\left(t_{\mathrm{InAs}}+t_{\mathrm{Mn}}\right)\right)$ was varied. It is obvious that the value $Y_{\mathrm{Mn}}$ is a technological parameter, which lets us operate the Mn content in the InMnAs layers from one run to another. The error of $Y_{\mathrm{Mn}}$ definition depends on the $t_{\mathrm{Mn}}$ value and decreases with increase in $t_{\mathrm{Mn}}$. The $Y_{\mathrm{Mn}}$ relative error comes to $30 \%$ and $5 \%$ for $Y_{\mathrm{Mn}}=0.05$ and $Y_{\mathrm{Mn}}=0.26$, respectively.

100-400 nm thick InMnAs layers were deposited on semiinsulating GaAs (100) substrates. During the deposition process the substrate was maintained at a temperature ranging from $280-330{ }^{\circ} \mathrm{C}$ in order to minimize [15] the sheet resistance $\left(R_{\mathrm{S}}\right)$.

The crystal quality and the phase composition were studied by x-ray diffraction on a DRON-4 diffractometer using $\mathrm{Cu} \mathrm{K}_{\alpha 1}$ radiation.

The electrical properties were derived from the Hall effect and magnetoresistance measurements at 77 and $300 \mathrm{~K}$ in magnetic fields $(H)$ up to 4000 Oe. We used Hall bar mesa structures fabricated by photolithography and etching to a depth of $\approx 1 \mu \mathrm{m}$ to obtain the following electrical characteristics: sheet resistance $R_{\mathrm{s}}$ (deduced from the $R_{x x}$ value), carrier concentration (deduced from $R_{x y}$ ) and Hall mobility $\mu_{\mathrm{H}}$. In the case of the normal Hall effect (when $R_{x y}$ is directly proportional to the magnetic field) the carrier concentration value is deduced immediately from $R_{x y}$. Otherwise (when $R_{x y}$ is a nonlinear function of magnetic field) the carrier concentration is obtained from the slope of the $R_{x y}$ versus the $H$ curve at maximum available magnetic fields.

The longitudinal magneto-optical Kerr effect (MOKE) measurements were performed at RT with the magnetic field applied in the sample plane.

Alternating gradient field magnetometry (AGFM) with a sensitivity of $10^{-8}$ emu was performed at $300 \mathrm{~K}$. The samples with InMnAs layers had a size of $6 \times 6 \times 0.4 \mathrm{~mm}^{3}$. A magnetic field was applied parallel to the sample surface.

FM resonance measurements were carried out on a Bruker ESP 300E spectrometer operating in the X-band $(9.38 \mathrm{GHz})$ at temperatures varying from 4 to $360 \mathrm{~K}$.

\section{Results and discussion}

The $\Theta / 2 \Theta$ x-ray diffraction curves for samples comprising heavily Mn-doped InAs layers that were produced in a flux of $\mathrm{AsH}_{3}+\mathrm{H}_{2}$ contain extra peaks in addition to the $\mathrm{GaAs}\left(\begin{array}{ll}0 & 0\end{array}\right)$ peak from the substrate and the $\operatorname{InAs}(002)$ peak from the layer near $31.6^{\circ}$ and $29.5^{\circ}$, respectively. The extra peaks at $42.3^{\circ}, 49.2^{\circ}$ and $31.9^{\circ}$ closely correspond to the calculated

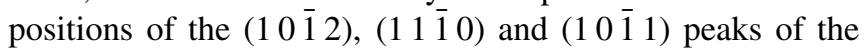
hexagonal MnAs phase, respectively. We can conclude that the InMnAs layers deposited at $T_{\mathrm{g}}=280-330^{\circ} \mathrm{C}$ and $Y_{\mathrm{Mn}}=$ $0.15-0.33$ exhibit fairly high crystal quality, but they are not single phase. X-ray diffraction spectra of the InMnAs layers produced without the $\mathrm{AsH}_{3}$ flux show the same peaks from the GaAs substrate and the InAs film, but no extra peaks from MnAs. However, a peak at $43.3^{\circ}$ emerges, which can be ascribed to the (3 30 ) diffraction of $\alpha-\mathrm{Mn}$. This fact suggests that in this case the incorporation of Mn into InAs takes place in the form of metallic clusters. Hence, the presence of arsine in the gas environment during the laser sputtering plays an important role in the formation of the crystal structure and phase composition of the deposited layers.

It is important that the presence of $\mathrm{AsH}_{3}$ in the gas flow has little effect on the electrical properties of the InAs layers undoped with Mn. Specifically, the $0.2 \mu \mathrm{m}$ thick InAs layer, grown without arsine at $320^{\circ} \mathrm{C}$, revealed a sheet resistance of $325 \Omega / \mathrm{sq}$, electron concentration $(n)$ of $5 \times 10^{17} \mathrm{~cm}^{-3}$ and electron Hall mobility $\left(\mu_{\mathrm{H}}\right)$ of $1820 \mathrm{~cm}^{2}(\mathrm{~V} \mathrm{~s})^{-1}$. A similar InAs layer, grown in a flux of $\mathrm{AsH}_{3}+\mathrm{H}_{2}$, had the same $R_{\mathrm{S}}$ value, $n=4.9 \times 10^{17} \mathrm{~cm}^{-3}$ and $\mu_{\mathrm{H}}=1960 \mathrm{~cm}^{2}(\mathrm{~V} \mathrm{~s})^{-1}$.

The InMnAs layers obtained without arsine flux show n-type conduction with an electron concentration of $\sim 3 \times 10^{16} \mathrm{~cm}^{-3}$, which implies a weak effect of the Mn doping, responsible for some compensation only. In all the above-mentioned cases (undoped InAs or InMnAs layers 

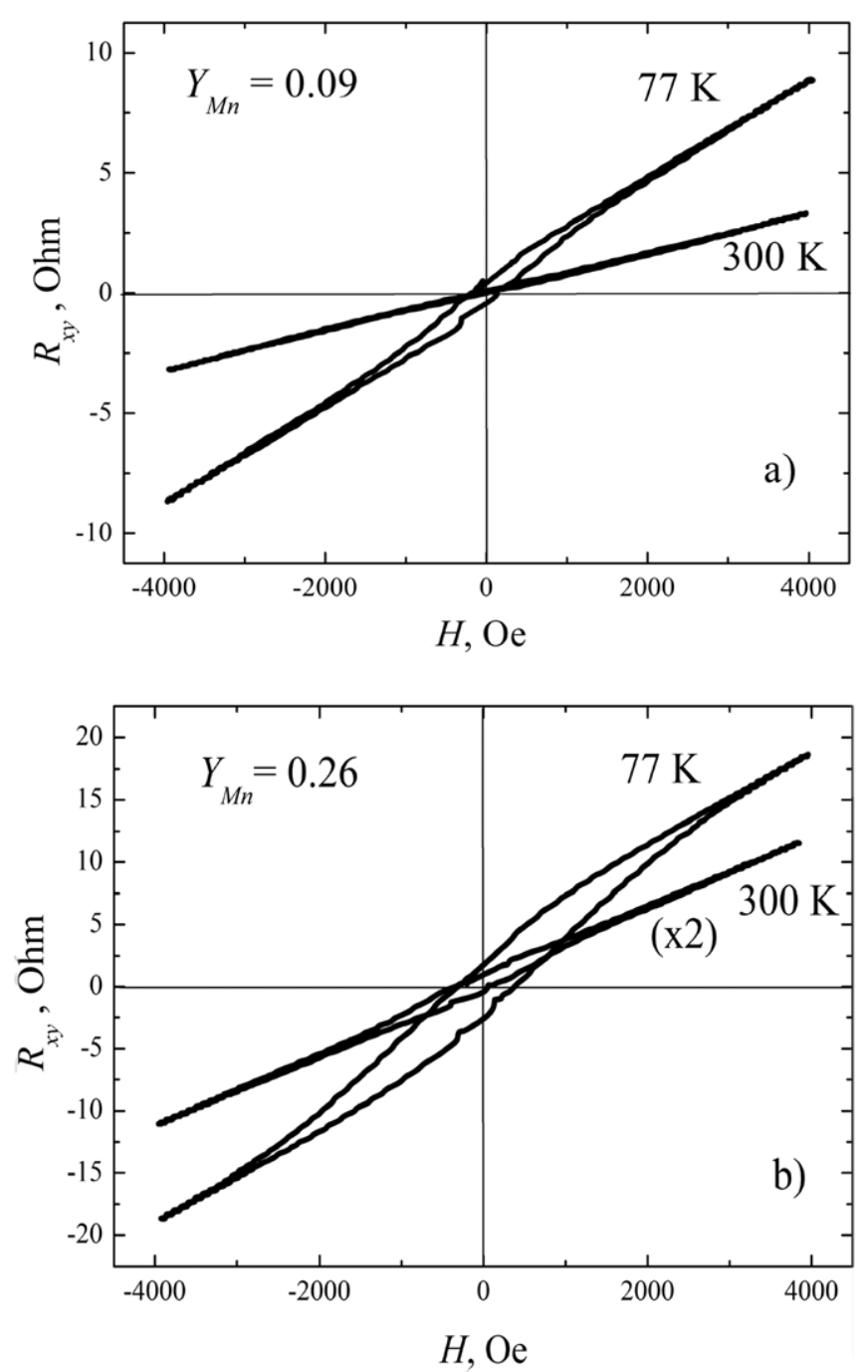

Figure 1. Hall resistance of InMnAs layers versus magnetic field at 77 and $300 \mathrm{~K}$ for two Mn concentrations.

grown in the absence of arsine) magnetic field dependences of the Hall resistance $\left(R_{x y}\right)$ are linear.

The InMnAs layers grown in the presence of arsine show p-type conduction for all used Mn concentrations $\left(Y_{\mathrm{Mn}}=\right.$ 0.05-0.33). Increasing $Y_{\mathrm{Mn}}$ within the range from 0.05 to 0.26 at $T_{\mathrm{g}}=320^{\circ} \mathrm{C}$ causes a monotonic enhancement of the $R_{\mathrm{S}}$ values: for RT measurements the product of $R_{\mathrm{S}}$ and layer thickness $(d)$ changes from $0.026 \Omega \mathrm{cm}\left(Y_{\mathrm{Mn}}=0.05\right)$ to $0.072 \Omega \mathrm{cm}\left(Y_{\mathrm{Mn}}=0.26\right)$. It was found that the InMnAs layers, deposited with an $\mathrm{AsH}_{3}$ flux, show an anomalous Hall effect both at $77 \mathrm{~K}$ and at $300 \mathrm{~K}$. Figure 1 depicts the magnetic field dependences of the Hall resistance $R_{x y}$ for two $Y_{\mathrm{Mn}}$ values.

For high Mn contents in the layer the Hall effect curve measured at $77 \mathrm{~K}$ (figure $1(b)$ ) shows a hysteresis loop with the coercive field $H_{\mathrm{c}} \sim 330 \mathrm{Oe}$ and saturation magnetic field $H_{\mathrm{s}} \approx 3000$ Oe. For RT measurements the hysteresis loop is also present ( $R_{x y}$ values were doubled for clarity), but with reduced values of $H_{\mathrm{c}}$ and $H_{\mathrm{s}}$.

For relatively small $\mathrm{Mn}$ contents the Hall effect curve shows a hysteresis loop only at $77 \mathrm{~K}$ (figure $1(a)$ ). We can see that the $H_{\mathrm{c}}$ and $H_{\mathrm{s}}$ values decrease as compared with the heavily doped layers down to 200 Oe and $\approx 2000$ Oe, respectively. We may note, however, that a small anomalous contribution is present even at $300 \mathrm{~K}$ for magnetic fields below 1000 Oe. It is surprising that in this case the sign of the anomalous term is negative. Insufficient accuracy of the Hall resistance definition near zero value of the magnetic field prevents a quantitative analysis of this curve. We would like to note only that previously [13] the negative sign of the anomalous Hall coefficient and superparamagnetic behaviour of MBE grown InMnAs layers were observed at $T<T_{\mathrm{c}} \approx$ $25 \mathrm{~K}$ for relatively low $(x \leqslant 0.03)$ concentrations of $\mathrm{Mn}$. Nevertheless, the authors [13] asserted that the origin of the negative sign for the anomalous Hall coefficient is unknown.

In our RT measurements the hysteresis loop arises only for $Y_{\mathrm{Mn}} \geqslant 0.13$.

The RT carrier concentration, simulated from the inclination of the Hall effect curve at maximum available magnetic fields, reaches $\sim 1 \times 10^{19} \mathrm{~cm}^{-3}$, with a hole mobility of $\approx 30 \mathrm{~cm}^{2}(\mathrm{~V} \mathrm{~s})^{-1}$. At $77 \mathrm{~K}$ the hole concentration decreases by a factor of $2-4$, and the mobility increases up to $\approx 60 \mathrm{~cm}^{2}(\mathrm{~V} \mathrm{~s})^{-1}$.

Calculation of charge carrier density within the limits of magnetic fields generally used by us (up to $4000 \mathrm{Oe}$ ) can underestimate the hole concentration in comparison with values for higher magnetic fields. The additional study, executed for the sample with $Y_{\mathrm{Mn}}=0.26$ in fields up to $1.6 \mathrm{~T}$, shows that the difference in values of the hole concentration amounts to about $30 \%$. An analogous comparison of hole densities for InMnAs layers, grown by MBE, was presented in [5], and the hole density difference had approximately the same $(\approx 30 \%)$ value.

The study of the RT longitudinal MOKE revealed a hysteresis loop in the dependences of the angle of polarization plane rotation on the magnetic field for InMnAs layers with $Y_{\mathrm{Mn}} \geqslant 0.17$

The InMnAs layers throughout the entire range of the Mn concentrations $\left(Y_{\mathrm{Mn}}=0.05-0.33\right)$ exhibit ferromagnetism as detected by magnetic measurements at RT. Figure 2 shows (curve 1) the magnetization versus the magnetic-field dependence, obtained by AGFM at $300 \mathrm{~K}$. It is seen that the magnetization consists of two contributions with different signs. The negative slope is related to the diamagnetic contribution of the GaAs substrate.

After extraction of this contribution (that is linear with magnetic field) we obtain a usual magnetization curve (curve 2 in figure 2) exhibiting a hysteresis loop. The magnetization versus magnetic-field curves are similar for the AGFM and MOKE measurements. Figure 3 shows the saturation magnetization $\left(M_{\mathrm{S}}\right)$ dependence on the $\mathrm{Mn}$ content in the InMnAs layers. The saturation magnetization increases with the $Y_{\mathrm{Mn}}$ value; however, according to the AGFM measurements, the coercive field $(\approx 300 \mathrm{Oe})$ is practically independent of the Mn concentration.

It should be noted that there is some difference in the thresholds for observation of FM properties from magnetization measurements $\left(Y_{\mathrm{Mn}}=0.05\right)$ and from the presence of a hysteresis loop on the magnetic field dependence of the Hall resistance $\left(Y_{\mathrm{Mn}}=0.13\right)$. The difference, apparently, can come from the fact that in the case of 


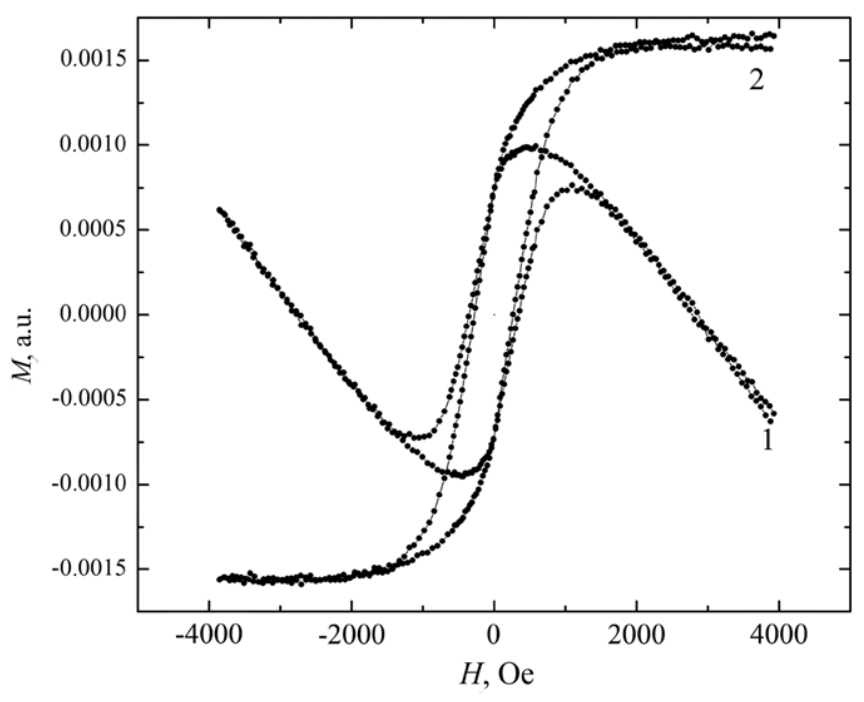

Figure 2. Magnetic field dependences of the magnetization for the sample containing an InMnAs layer with $Y_{\mathrm{Mn}}=0.13: 1$ - as measured by AGFM; 2 -after extraction of the diamagnetic contribution. $T_{\mathrm{m}}=300 \mathrm{~K}$.

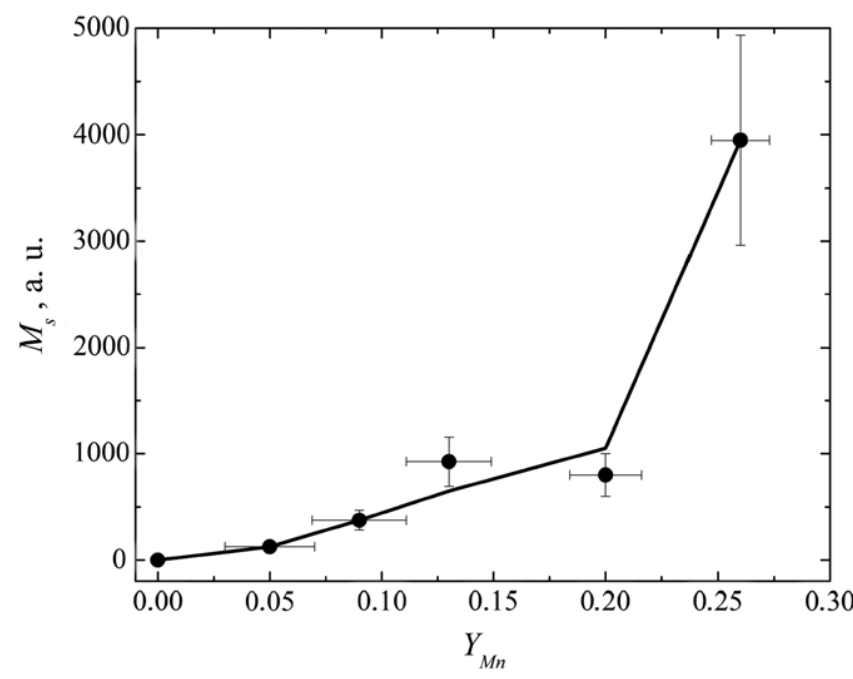

Figure 3. Dependence of the saturation magnetization, determined by the AGFM technique, on the Mn content in the InMnAs layers. $T_{\mathrm{m}}=300 \mathrm{~K}$.

magnetization measurements the response was measured from all FM phases, but the appearance of a nonlinear magnetic field dependence of Hall resistance with a hysteresis loop can be a consequence of spin polarized carrier transport. Our samples contain at least two phases: p-InMnAs matrix and semimetal MnAs inclusions. As was shown in our previous work [15], the conductivity of InMnAs samples demonstrates semiconductor behaviour, i.e. we can assume that transport of carriers occurs in the p-InMnAs matrix. Largest concentrations of carriers correspond to the samples with $Y_{\mathrm{Mn}}=0.09-0.13$ and smallest concentration to the sample with $Y_{\mathrm{Mn}}=0.05$. We can suppose that in the layer with $Y_{\mathrm{Mn}}=0.05$ the hole concentration is too small $\left(\approx 1 \times 10^{18} \mathrm{~cm}^{-3}\right)$ to realize ferromagnetism in the p-InMnAs matrix, but there are MnAs clusters which produce FM response in AGFM magnetization measurements.

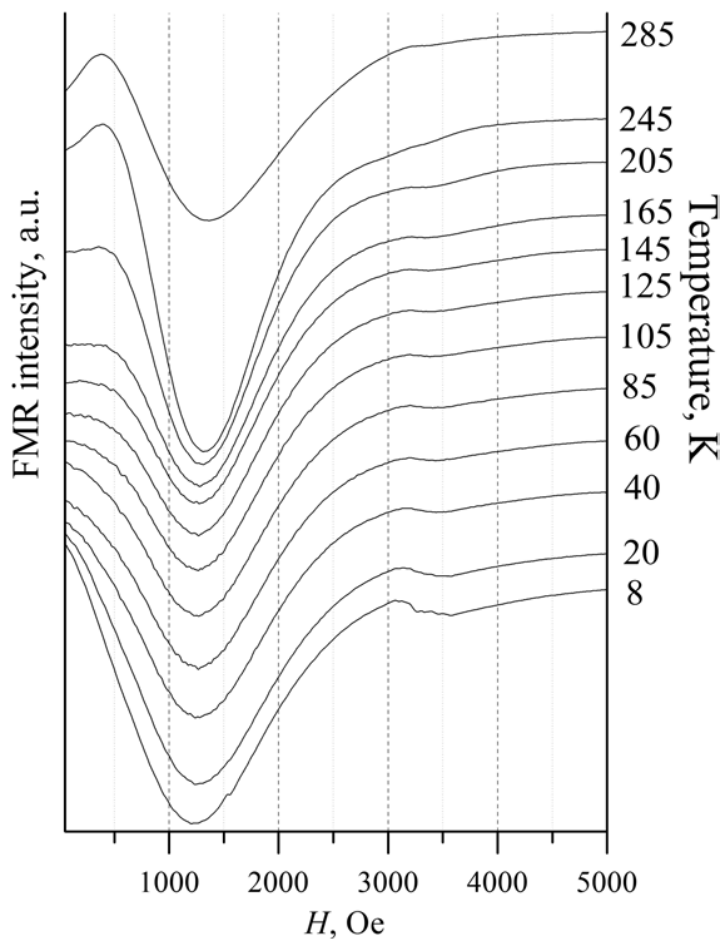

Figure 4. Temperature dependence of the FMR spectra of sample with $Y_{\mathrm{Mn}}=0.20$ for the orientation of the magnetic field perpendicular to the sample plane. The spectra are vertically shifted for clarity.

FMR measurements detected a broad absorption band that did not emerge in the reference InAs samples; its intensity grows with increasing $Y_{\mathrm{Mn}}$. The signal reduces rapidly at temperatures above $265 \mathrm{~K}$; however, the line is still observable at $300 \mathrm{~K}$ and completely disappears at $330 \mathrm{~K}$. Thus, the Curie temperature $T_{\mathrm{C}}$ of the corresponding magnetic phase is slightly above RT (the FMR is usually observed even at the paramagnetic side of $T_{\mathrm{C}}$ [16]). This value is quite compatible with $T_{\mathrm{C}}$ of $\alpha$-MnAs (313 K [17]). The resonance field position and width are found to be a function of the measurement temperature (see, e.g., figure 4) and of the sample orientation in the applied magnetic field. However, the angular dependence is weak. The resonance field is always below that of the paramagnetic resonance value corresponding to $g=2$ and approaches the latter with increasing temperature. Simultaneously, the line width decreases. This behaviour is typical of magnetic nanoparticles randomly embedded in a solid matrix [18]. The line width is determined by the spread of the resonance fields of the individual nanoparticles caused by the random distribution of their easy magnetization axes. With the temperature approaching the Curie point, the magnetocrystalline anisotropy disappears, and the line gets narrower. The weak anisotropy of the line position can be explained by a residual alignment of the nanoparticle axes in the host matrix. However, the unique strong angular dependence observed for hexagonal MnAs nanoclusters epitaxially aligned in GaAs [19] or Si [20] has not been observed. The interpretation of the FMR results is complicated by the fact that in our measurements the magnetization is probably not saturated due to the very high 
saturation magnetization fields observed, for example, by AHE (figure 1). The FMR will be analysed in detail in a forthcoming publication.

\section{Conclusions}

We can conclude that InMnAs layers, obtained by laser ablation in an arsine flux, show ferromagnetism up to $300 \mathrm{~K}$. We demonstrated for the first time an anomalous Hall effect in a semiconductor (III, Mn)As at RT. The Hall data are consistent with the FMR observations. Thus, the InMnAs layers can be used as RT FM components, e.g. in spin light-emitting diodes.

\section{Acknowledgments}

This work was supported by RFBR (projects 08-0200548 and 08-02-97038), Ministry of Education of Russian Federation (project 2.2.2.2.4737), and FTC of Portugal (project POCI/FIS/61462/2004). One of the authors (YuAD) is grateful to student $\mathrm{A} \mathrm{V}$ Alaferdov for the help in data treatment.

\section{References}

[1] Munekata H, Ohno H, von Molnar S, Segmuller A, Chang L L and Esaki L 1989 Phys. Rev. Lett. 631849

[2] Onho H, Munekata H, Penney T, von Molnar S and Chang L L 1992 Phys. Rev. Lett. 682664

[3] Iye Y, Oiwa A, Endo A, Katsumoto S, Matsukura F, Shen A, Onho H and Munekata H 1999 Mater. Sci. Eng. B 6388

[4] Matsukura F, Chiba D, Omiya T, Abe E, Dietl T, Onho Y, Ohtani K and Onho H 2002 Physica E 12351
[5] Schallenberg T and Munekata H 2007 J. Cryst. Growth 301-302 623

[6] Blattner A J, Lensch J and Wessels B W 2001 J. Electron. Mater. 301408

[7] Blattner A J, Prabhumirashi P L, Dravid V P and Wessels B W 2003 J. Cryst. Growth 2598

[8] Blattner A J and Wessels B W 2004 Appl. Surf. Sci. 221155

[9] Soo Y L, Kim S, Kao Y H, Blattner A J, Wessels B W, Khalid S, Hanke C S and Kao C-C 2004 Appl. Phys. Lett. 84481

[10] May S J, Blattner A J and Wessels B W 2003 Physica B 340-342 870

[11] Rylkov V V, Lagutin A S, Aronzon B A, Podolskii V V, Lesnikov V P, Goiran M, Galibert J, Raquet B and Leotin J 2006 arXiv: Cond-Mat 0612641

[12] Munekata H, Abe T, Koshihara S, Oiwa A, Hirasawa M, Katsumoto S, Iye Y, Urano C and Takagi H 1997 J. Appl. Phys. 814862

[13] Matsukura F, Chiba D, Omiya T, Abe E, Dietl T, Ohno Y, Ohtani K and Ohno H 2002 Physica E 12351

[14] Zvonkov B N, Vikhrova O V, Danilov Yu A, Demidov E S, Demina P B, Dorokhin M V, Drozdov Yu N, Podolskii V V and Sapozhnikov M V 2008 J. Opt. Technol. 75389

[15] Vikhrova O V, Danilov Yu A, Zvonkov B N, Kudrin A V, Podolskii V V, Drozdov Yu N, Sapozhnikov M V, Moura C, Vasilevskiy M I and Temiryazeva M P 2008 Phys. Solid State $\mathbf{5 0} 52$

[16] Farle M 1998 Rep. Prog. Phys. 61755

[17] Okamoto H 1989 Bull. Alloy Phase Diagr. 10549

[18] For a review see, e.g. Zakeri K H, Kebe T H, Lindner J, Antoniak C, Farle M, Lenz K, Tolinski T and Baberschke K 2006 Phase Transit. 79793 and references therein

[19] Hartmanna Th, Lampalzer M, Klar P J, Stolz W, Heimbrodt W, Krug von Nidda H-A, Loidl A and Svistov L 2002 Physica E 13572

[20] Sobolev N A, Oliveira M A, Amaral V S, Neves A, Carmo M C, Wesch W, Picht O, Wendler E, Kaiser U and Heinrich J 2006 Mater. Sci. Eng. B 126148 